\title{
Publisher Correction: Hierarchical self-entangled carbon nanotube tube networks
}

\author{
Fabian Schütt [1] ${ }^{1}$, Stefano Signetti [i ${ }^{2}$, Helge Krüger ${ }^{1}$, Sarah Röder ${ }^{1}$, Daria Smazna ${ }^{1}$, Sören Kaps ${ }^{1}$, \\ Stanislav N. Gorb ${ }^{3}$, Yogendra Kumar Mishra (1) ${ }^{1}$, Nicola M. Pugno (iD) ${ }^{2,4,5}$ \& Rainer Adelung ${ }^{1}$
}

Correction to: Nature Communications https://doi.org/10.1038/s41467-017-01324-7, Article published online 31 October 2017

The original version of this Article was missing the ORCID ID of Professor Nicola Pugno.

Also in the original version of this Article, the third to last sentence of the fourth paragraph of the Results incorrectly read "However, the stepwise addition of CNTs increases the self-entanglement and thereby the compressive strength value as well as the Young's modulus (up to $2.5 \mathrm{MPa}$ (normalized by density 6.4) and $24.5 \mathrm{MPa}$ (normalized by density $62 \mathrm{MPa} \mathrm{cm}^{3} \mathrm{~g}^{-1}$ )." The correct version adds the units "MPa cm $\mathrm{g}^{-1}$ " to " 6.4 ".

Finally, in the original version of this Article, the $y$-axis label of Fig. $3 \mathrm{f}$ incorrectly read "Comp. strengthy". The new version corrects that to "Comp. Strength".

These errors have now been corrected in both the PDF and the HTML versions of the Article.

Published online: 09 January 2018

\begin{abstract}
(c) (i)
Open Access This article is licensed under a Creative Commons Attribution 4.0 International License, which permits use, sharing, adaptation, distribution and reproduction in any medium or format, as long as you give appropriate credit to the original author(s) and the source, provide a link to the Creative Commons license, and indicate if changes were made. The images or other third party material in this article are included in the article's Creative Commons license, unless indicated otherwise in a credit line to the material. If material is not included in the article's Creative Commons license and your intended use is not permitted by statutory regulation or exceeds the permitted use, you will need to obtain permission directly from the copyright holder. To view a copy of this license, visit http://creativecommons.org/licenses/by/4.0/.
\end{abstract}

(C) The Author(s) 2018

\footnotetext{
${ }^{1}$ Functional Nanomaterials, Institute for Materials Science, Kiel University, Kaiserstr. 2, 24143 Kiel, Germany. ${ }^{2}$ Laboratory of Bio-Inspired and Graphene Nanomechanics, Department of Civil, Environmental and Mechanical Engineering, University of Trento, via Mesiano 77, I-38123 Trento, Italy. ${ }^{3}$ Functional Morphology and Biomechanics, Zoological Institute, Kiel University, Am Botanischen Garten 1-9, 24098 Kiel, Germany. ${ }^{4}$ Ket Lab, Edoardo Amaldi Foundation, Italian Space Agency, Via del Politecnico snc, 00133 Rome, Italy. ${ }^{5}$ School of Engineering and Materials Science, Queen Mary University of London, Mile End Road, E1 4NS London, UK. Correspondence and requests for materials should be addressed to F.S. (email: fas@tf.uni-kiel.de) or to N.M.P. (email: nicola.pugno@unitn.it) or to R.A. (email: ra@tf.uni-kiel.de)
} 